Annuaire suisse de politique de développement

27-2 | 2008

Migration et développement: un mariage arrangé

\title{
La circulation des compétences, un enjeu pour le développement
}

Jean-Baptiste Meyer

\section{(2) OpenEdition}

1 Journals

Édition électronique

URL : http://journals.openedition.org/aspd/167

DOI : 10.4000/aspd.167

ISSN : 1663-9669

Éditeur

Institut de hautes études internationales et du développement

Édition imprimée

Date de publication : 1 décembre 2008

Pagination : 53-67

ISBN : 978-2-940415-07-6

ISSN : 1660-5934

\section{Référence électronique}

Jean-Baptiste Meyer, «La circulation des compétences, un enjeu pour le développement », Annuaire suisse de politique de développement [En ligne], 27-2 | 2008, mis en ligne le 19 mars 2010, consulté le 08 septembre 2020. URL : http://journals.openedition.org/aspd/167 ; DOI : https://doi.org/10.4000/ aspd. 167 


\section{La circulation des compétences, un enjeu pour le développement}

Jean-Baptiste Meyer*

\section{Introduction}

Dans l'étude des migrations et des mobilités, le paradigme circulatoire s'est substitué à celui de la fuite des cerveaux (brain drain). Le changement, réalisé au tournant du millénaire, consacre une nouvelle vision, globalisée plus que statocentrique, du mouvement. Une connotation positive y est souvent attachée: les compétences se meuvent sur la planète d'une façon moins unilatérale que par le passé. Les flux Sud-Nord des détenteurs de ces compétences peuvent notamment être compensés par des retours, par des connexions à distance, par des mises à disposition occasionnelles, au bénéfice des pays d'origine. Ces derniers peuvent ainsi capitaliser des réseaux et des capacités du Nord, à travers leurs ressortissants expatriés, en faisant l'économie de créations coûteuses (brain gain).

Les flux migratoires d'orientation Sud-Nord surtout - des pays en développement vers les Etats-Unis et l'Europe - mobilisent des nombres importants et constituent des populations respectables dans les pays d'accueil. Il en résulte aujourd'hui la constitution de groupes de professionnels provenant d'un même pays et localisés dans d'autres, pour exercer des activités où leurs connaissances sont cruciales. Ces groupes sont ici dénommés «diasporas du savoir» ou diaspora knowledge networks. En effet, leurs membres revendiquent une même origine passée, bien qu'ils vivent dans la dispersion au temps présent. Leurs compétences et leurs activités cognitives fondent leur appartenance à ces groupes, souvent formalisés en associations avec des objectifs de développement avérés. Cette conjonction de traits - origine commune, dispersion spatiale, statut intellectuel, objectif développemental - en fait bien d'authentiques diasporas, mais avec une base sociale particulière. Le lien social entre individus et groupes constitutifs éparses n'est pas construit seulement par l'histoire et l'expérience partagées, mais par une unité de compétence et de projet.

La particularité des diasporas du savoir tient non seulement à ces traits spécifiques, mais également à leur apparition récente. La première partie de ce texte révèle comment ces groupes diasporiques ont peu à peu pris une autonomie qui donne un sens nouveau à la circulation des compétences. La partie suivante s'attache à décrire ce que sont ces diasporas en termes d'organisation, de liens et d'actions, à partir de quelques études de cas. Puis la troisième partie étend ces constats empiriques limités à de multiples cas identifiés dans le monde. Enfin, la dernière partie synthétise, à partir des expériences diasporiques examinées, une forme de modélisation des pratiques relationnelles fécondes pour le développement.

* Directeur de recherche à l'Institut de recherche pour le développement (IRD, France). 
Durant les décennies 1960-1980, la perception de la mobilité des personnes qualifiées s'est effectuée dans le cadre du paradigme de la «fuite des cerveaux». Cette dénomination est apparue à la fin des années 1950 puis a été entérinée en 1963 dans un document officiel de la Royal Society ${ }^{1}$. Les compétences s'exilaient apparemment de façon permanente ou durable, unilatérale et alignée sur un axe Sud-Nord où leur motion était supposément déterminée par le simple différentiel de revenus, de conditions de vie et d'exercice professionnel. Dans les pays d'origine, l'absence ou la précarité de communautés et d'infrastructures scientifiques et techniques, académiques ou industrielles sophistiquées excluaient $a$ priori tout retour substantiel de talents formés à l'étranger. Leur inadéquation pour des tâches d'utilisation locale ainsi que le faible niveau des revenus réservaient peu de possibilités d'insertion à ces personnes. Beaucoup de programmes de retour impulsés par les gouvernements des pays de départ échouèrent ainsi souvent, avec de faibles proportions de personnes revenant effectivement, au regard du nombre des départs. Souvent partis individuellement ou avec un suivi institutionnel minimal, les expatriés immergés dans le tissu professionnel du pays d'accueil entretenaient rarement des relations systématiques soutenues avec leurs compatriotes, à l'extérieur ou au pays.

La décennie 1990 voit l'apparition de nombreux réseaux diasporiques de personnes hautement qualifiées. Cette émergence coïncide complètement avec celle des réseaux de télécommunication médiatisée par ordinateur: Arpanet, Bitnet et Internet. Il ne s'agit guère d'un fait du hasard ni d'un déterminisme technique absolu, mais bel et bien d'une conjonction sociotechnique. En effet, ces modalités de communication sont directement constitutives des réseaux. Le réseau Caldas des chercheurs et ingénieurs colombiens à l'étranger, par exemple, a recruté ses membres dispersés dans 25 pays, à travers leur identification, leur localisation et leur mobilisation par Internet. Des appels à la cantonade puis des recherches ciblées sur les newsgroups ont permis aux expatriés colombiens de se compter, d'échanger, de décider de se constituer en association formelle puis de faire passer le message aux autres membres potentiels ${ }^{2}$. Enfin, une fois ces membres identifiés, localisés et mobilisés, la liste R-Caldas, qui permet à tous ceux qui sont connectés de recevoir et d'envoyer des messages, constitue le lien collectif entre toutes ces parties dispersées. Il n'est pas anodin de constater que ce ferment unitaire constitué par la communication électronique a d'ailleurs affecté négativement le réseau, un temps après la fusion initiale. Une fois inscrits sur la liste, de nombreux membres ont progressivement fait défection, par lassitude ou irritation de recevoir de façon quotidienne des messages sans rapport avec leur travail et de peu d'utilité. Ce fait rappelle une originalité de la structure diasporique, modulaire plus que communautaire, fondée sur un lien réticulaire activé de façon plus intermittente que permanente. Les groupes de la diaspora sont, en effet, normalement en relation de façon sporadique et vivent des vies séparées bien que la communication les associe à l'occasion.

1 J. Gaillard et A.-M. Gaillard, Les enjeux des migrations scientifiques internationales. De la quête du savoir à la circulation des compétences, Paris, L'Harmattan, 1997, p. 47.

2 J. Granés and J.-B. Meyer, «Globalization of the National Scientific Community through Electronic List: Lessons and Prospects from a Case Study in Colombia», in J. Charum and J.-B. Meyer (eds.), International Scientific Migrations Today, CD-Rom, Paris, Editions IRD, 2000. 
Au tournant du millénaire, la mobilité des personnes qualifiées a pris une nouvelle ampleur. C'était l'époque de la «nouvelle économie» et de la bulle Internet, avec un pôle d'activité largement centré sur l'Amérique du Nord et, dans une moindre mesure, sur l'Europe de l'Ouest. Ces zones attractrices ont généré des flux importants de main-d'œuvre qualifiée depuis les régions du monde en développement. Cette accélération de la mobilité, fruit d'une véritable spéculation symbolique en même temps qu'économique, a réveillé de nombreuses inquiétudes. Une recension des discours politiques et médiatiques fait état de maintes occurrences alarmistes, prospectives ou stratégiques, invoquant le phénomène dans des termes aussi éloquents que ceux de «fuite des cerveaux», «évasion de capital humain», «hémorragie de ressources compétitives», «guerre pour l'obtention des talents », etc. ${ }^{3}$ Ces références imagées dénotent une charge sémantique importante.

A l'orée du XXI ${ }^{\mathrm{e}}$ siècle, la mobilité des personnes qualifiées est redevenue, peutêtre plus encore que quarante ans auparavant lors de l'émergence du brain drain, un enjeu politique international significatif. Dans les conflits d'interprétation dont ce dernier est l'objet, l'option diaspora constitue une ressource discursive non négligeable. Elle est mobilisée comme instrument possible de résorption du déficit de ressources humaines par leur récupération à distance, comme preuve d'une compensation automatique offerte par les pays du Nord recevant nombre d'expatriés du Sud, comme alibi pour des pays du Sud inactifs dans le développement de leurs ressources humaines locales, comme cache-misère pour des agences intergouvernementales trop heureuses d'interpréter les pertes liées aux asymétries Nord-Sud comme des gains potentiels...

L'apparition de réseaux organisés de chercheurs et d'ingénieurs expatriés à un moment où la prise de conscience de l'émigration de ce type de personnel s'accroissait a suscité un intérêt certain. Au-delà des interprétations diverses, elle a révélé des formes nouvelles de migration et de socialisation, ouvrant des perspectives inédites sur les relations transnationales et les liens scientifiques et techniques.

\section{Les diasporas du savoir}

Les diasporas du savoir sont généralement organisées sur une base associative et souvent animées par un même objectif de contribuer à distance au développement des lieux d'origine. Leurs actions sont multiples et vont de l'échange d'information spécialisée avec les collègues restés au pays jusqu'au transfert de technologie, en passant par le tutorat d'étudiants avancés dans les laboratoires du Nord où sont insérés les expatriés et par la participation ponctuelle de ces derniers à des sessions de formation dans les universités d'origine ou à la réalisation de projets de recherche ou de développement conjoints, comportant des phases tantôt virtuelles, tantôt concrètes. Plusieurs centaines de ces réseaux scientifiques diasporiques formels ont été identifiés (voir plus bas). La taille,

3 L. Lowell, Skilled Labour Migration from Developing Countries: Annotated Bibliography, International Migration Papers, $n^{\circ}$ 56, Geneva, International Labour Office, 2002. A.M. Findlay and E. Stewart, Skilled Labour Migration from Developing Countries: Annotated Bibliography, International Migration Papers, $n^{\circ}$ 55, Geneva, International Labour Office, 2002. 
l'éventail d'activités, le nombre de pays couverts, l'organisation, la pérennité et les objectifs de ces réseaux varient grandement.

Le réseau Caldas possédait quelques centaines d'inscrits dans plusieurs «nœuds» locaux aux Etats-Unis et en Europe. Le réseau SANSA (South African Network of Skills Abroad) rassemble plus de 2500 membres répartis dans 65 pays du monde. Les nombreuses associations indiennes et chinoises regroupant plusieurs milliers de membres (American Society of Engineers of Indian Origin, Chinese Association of Science and Technology-USA, par exemple) sont particulièrement actives aux Etats-Unis avec la bénédiction, et parfois le soutien actif, de leur pays d'origine. Les Marocains ou les Tunisiens ont également plusieurs associations d'expatriés hautement qualifiés, certaines francophones et d'autres présentes sur les campus nord-américains (Savoirs et Développement, Tunisian Scientific Consortium, Association des chercheurs enseignants tunisiens de France). Les biologistes algériens expatriés ont fondé un réseau disciplinaire (Algebio).

Ces réseaux d'expatriés diffèrent par leur nature: scientifique, technique, institutionnelle, professionnelle, financière. Ce qui les rend précieux pour leurs pays d'origine, ce sont les compétences déployées dans des activités cognitives et rendues disponibles pour ces pays, mais aussi l'accès à des équipements et capacités qui y sont absents, de même que le statut, la crédibilité et la reconnaissance dérivant de l'appartenance à des laboratoires ou entreprises de renommée mondiale et ainsi prêtés à une entité périphérique. La participation à de grands programmes internationaux dotés de financements importants et l'introduction à des marchés spécialisés, tout particulièrement à travers l'adoption de procédures, de standards et de contrôles de qualité, se révèlent des apports cruciaux ${ }^{4}$. Sans l'entremise de ces acteurs diasporiques, la pénétration de ces consortiums et de ces marchés très fermés ne serait guère possible.

Les constats empiriques montrent qu'un nombre substantiel de personnes hautement qualifiées sont désireuses d'œuvrer pour leur pays d'origine. Les motivations individuelles mentionnées apparaissent diverses: sentiment de culpabilité d'avoir abandonné les siens et fait fortune au loin, engagement militant ou souvenirs sentimentaux, opportunités pratiques de rester en contact avec des proches à travers les réseaux formels, expectatives de développements professionnels, expansion sociale ou entrepreneuriale, occasion d'établir des connexions internationales et de recevoir le soutien d'agences de coopération, etc. Quelles que soient les raisons, les membres de la diaspora sont généralement sensibles à la situation du pays, ouverts et souvent disponibles pour une éventuelle coopération. Bien que seule une fraction puisse parfois répondre à l'appel du pays d'origine, des sondages occasionnels parmi des expatriés qui n'avaient pas fait l'effort de se reconnecter ont révélé de fait moins un désintérêt

4 J. Granés y A. Morales, «Las Potencialidades y Limitaciones de la Red Caldas de Investigadores Colombianos en el Exterior: Los Proyectos Internacionales Conjuntos: Un Estudio de Casos», in J. Charum y J.-B. Meyer (coordinadores), Hacer Ciencia en un Mundo Globalizado: La Diáspora Científica Colombiana en Perspectiva, Santafé de Bogotá, TM Editores, 1998. J. Johnson, «S\&T Resources and Programs in the United States for Networking with Developing Countries», in R. Barré, V. Hernandez, J.-B. Meyer et D. Vinck, Diasporas scientifiques/Scientific Diasporas, Paris, Editions IRD, 2003. R. Lucas, International Migration Regimes and Economic Development, Stockholm, Expert Group on Development Issues (EGDI), 2004. 
que la simple ignorance ou inconscience des tentatives du pays d'origine. Le désir de relation provient donc généralement des deux côtés: des expatriés et des communautés d'origine.

Au-delà des dispositions individuelles, cependant, il y a des processus collectifs qui œuvrent à la constitution des réseaux de diasporas de la connaissance. L'expression identitaire y est essentielle ${ }^{5}$. Leurs membres se réclament d'une même origine, d'une trajectoire migratoire identique ou comparable et d'un exercice commun (celui d'activités hautement qualifiées). Nos résultats sur les réseaux colombiens et sud-africains montrent que ces expressions identitaires sont profondément construites par les acteurs eux-mêmes au moment où ils intègrent le réseau ${ }^{6}$. Elles ne le précèdent pas et ne lui survivent guère. Le réseau est ainsi performatif de cette identité; il n'en est pas le produit dérivé. Dans cette approche constructiviste de la formation de l'identité, le rôle des technologies de la communication est crucial.

L'identification des professionnels expatriés à leur pays d'origine est un produit de leur mise en réseau au travers des diasporas de la connaissance. Cependant, elle correspond aussi à un phénomène plutôt classique décrit par la sociologie. Le marché du travail, traditionnellement régulé et bordé par les frontières territoriales, est un puissant creuset des identités nationales ${ }^{7}$. Il n'est alors guère surprenant que la reconnaissance par les pays d'origine d'aptitudes professionnelles, cruciales dans la formation d'identités professionnelles, génère en retour une extension de celles-ci à la sphère nationale d'origine ${ }^{8}$. C'est une ré-identification sous motifs professionnels. En d'autres termes, c'est au moment où les pays d'origine insistent sur l'importance des catégories hautement qualifiées dans la construction de sociétés basées sur les savoirs que les migrants qui en relèvent s'y identifient de façon inédite. Ce constat pointe un fait important: les processus d'identification sont dynamiques et liés à l'action. Ils sont liés à l'action professionnelle actuelle plutôt qu'à des survivances culturelles passives. C'est particulièrement le cas pour les pratiques cognitives professionnelles. La production et le développement de connaissances sont, en effet, des activités dans lesquelles les processus identitaires à travers la projection collective dans l'avenir sont fondamentaux. Les nouvelles diasporas de chercheurs et d'ingénieurs ont contribué à illuminer ce phénomène d'une façon inattendue ${ }^{9}$. La prise de conscience croissante de l'importance de la connaissance dans les processus de développement, stimulée par l'émergence des systèmes nationaux d'innovation en même temps que l'ouverture de nombreux pays du Sud durant les années 1990 (Argentine, Chili, Chine, Colombie, Inde), a suscité un processus de ré-intégration active entre la diaspora intellectuelle et les pays d'origine.

5 J. Brinkerhoff, «Diasporas, Mobilization Factors and Policy Options», in C. Wescott and J. Brinkerhoff (eds.), Converting Migration Drains into Gains: Harnessing the Resources of Overseas Professionnals, Manila, Asian Development Bank, 2006, pp. 127-153.

6 J.-B. Meyer et al., «Turning Brain Drain into Brain Gain: The Colombian Experience of the Diaspora Option», Science, Technology and Society, vol. 2, n 2, 1997, pp. 285-317. J.-B. Meyer, D. Kaplan and J. Charum, «Scientific Nomadism and the New Geopolitics of Knowledge», International Social Sciences Journal/Revue internationale des sciences sociales, $\mathrm{n}^{\circ}$ 168, 2001, pp. 341-354.

E. Gellner, Nations and Nationalism, Oxford, Basil Blackwell, 1983.

8 C. Dubar, La socialisation: construction des identités sociales et professionnelles, Paris, Armand Colin, 1991.

9 J.-B. Meyer, «Savoirs, diasporas et identités projectives », Hermès, n 40, 2004, pp. 350-354. 
Cette nouvelle approche intégrative est parfois perçue comme une politique étatique, authentique et inédite ${ }^{10}$.

Cette intégration des marges diasporiques dans les politiques des Etats-nations apparaît comme une pratique de plus en plus courante et légitime, à l'instar de zones géographiques frontalières où l'échange intensif est propice à une nouvelle fertilisation croisée.

\section{Controverses autour de la mobilité et des diasporas}

Le débat autour de la mobilité des personnes qualifiées et de ses conséquences, nuisibles ou bénéfiques - brain drain vs brain gain - demeure d'actualité ${ }^{11}$. Dans ce contexte, plusieurs auteurs ont récemment mis en question l'existence et la consistance des réseaux diasporiques scientifiques et techniques ainsi que leurs effets sur les pays d'origine ${ }^{12}$. Après quelques examens empiriques d'un certain nombre de réseaux, pays ou régions, leurs doutes ou critiques se cristallisent autour des points suivants :

- L'amplitude du phénomène: combien y a-t-il de réseaux dans le monde? Ceux identifiés sont-ils quelques cas isolés ou un échantillon représentatif? Ces exemples d'identification des expatriés sont-ils une exception ou une nouvelle forme de socialisation internationale?

- La durabilité des associations d'expatriés: sont-elles équipées pour tenir plus que quelques mois ou années? Comme regroupements temporaires de personnes mobiles, leurs membres sont-ils suffisamment stables pour assurer une certaine continuité? Peut-on la vérifier dans un certain nombre de cas?

- L'interprétation de la visibilité sur Internet et la représentativité de la présence sur le web: les sites reflètent-ils des associations aux activités réelles et productives? Certains sites, toujours visibles mais peu actifs, ne sont-ils pas des étoiles mortes envoyant la lumière d'une vie déjà éteinte? N'y a-t-il pas extrapolation exagérée à déduire de quelques fenêtres bien présentées une véritable solution de l'exode des compétences?

- L'apport des réseaux au développement scientifique, technique et économique des pays d'origine: ces réseaux peuvent-ils réellement suppléer à l'absence de communauté académique locale ? Est-il possible de mener un travail conjoint effectif en dépit du manque de ressources, d'équipements et d'homologues sur place? Peut-on attribuer les développements spectaculaires de secteurs informatiques et de haute technologie en Asie à l'apport de la diaspora et non plutôt à des facteurs purement locaux?

10 A. Gamlen, The Brain Drain Is Dead: Long Live the New Zealand Diaspora, COMPAS Working Paper, $\mathrm{n}^{\mathrm{0}}$ 10, Oxford, Centre on Migration, Policy and Society (COMPAS), Oxford University, 2005.

11 F. Docquier and A. Marfouk, Measuring the International Mobility of Skilled Workers (1990-2000), World Bank Policy Research Working Paper, $\mathrm{n}^{\circ}$ 3381, Washington, DC, World Bank, 2004. J.-C. Dumont and G. Lemaître, «Beyond the Headlines: New Evidence on the Brain Drain», La Revue économique, vol. 56, $\mathrm{n}^{\circ}$ 6, 2005, pp. 1275-1299.

12 J. Gaillard and A.-M. Gaillard, «Can the Scientific Diaspora Save African Science?», 2003, disponible sur le site du Science and Development Network (Scidev), <http://www.scidev.net>. L. Lowell and S. Gerova, Diasporas and Economic Development: State of Knowledge, Washington, DC, World Bank, 2004. R. Lucas, op. cit. 
Aujourd'hui, les résultats empiriques relatifs aux réseaux de diasporas hautement qualifiées permettent de répondre à bon nombre de ces questions. Ces données proviennent de l'analyse systématique de plus d'une centaine de sites Internet et de l'observation historique précise des cas de transfert et de circulation entre l'Asie et l'Amérique du Nord.

Tout d'abord, cinq recensements, effectués par des équipes différentes à divers moments, ont permis de confirmer l'existence des réseaux comme représentatifs d'un phénomène général :

• 41 associations émanant de pays développés et en développement ${ }^{13}$;

๑ 106 réseaux identifiés comme étant en lien avec des pays en développement exclusivement ${ }^{14}$;

๑ 61 réseaux de pays développés et en développement ${ }^{15}$;

- 158 réseaux repérés pour des pays en développement ${ }^{16}$;

口 191 réseaux de pays développés et en développement ${ }^{17}$.

En l'absence de tels chiffres, la réalité du phénomène diasporique, la représentativité des quelques cas identifiés au cours des années 1990, restait sujette à caution. Le fait de pouvoir montrer qu'il existe des dizaines, voire des centaines de cas interdit de minimiser la consistance de l'option diaspora et consolide la prééminence de l'approche circulatoire.

Les différents recensements ont permis d'affiner progressivement les techniques de recherche sur Internet et de mettre au jour un nombre très important de réseaux préalablement peu visibles. Pourtant, compte tenu du fait que les recherches par Internet ne garantissent jamais que l'on ait épuisé toutes les réponses possibles, on peut légitimement penser que bien des réseaux sont encore non recensés à ce jour et que leur nombre dépasse largement les 150 identifiés.

Cette hypothèse est même devenue une certitude depuis la publication d'une étude récente sur les réseaux chinois. Là où notre examen systématique avait dégagé la présence de seulement 12 réseaux chinois sur Internet, la recherche chinoise, utilisant des mots clés en langues locales, en fait apparaître $200^{18}$. Cette étude, commandée par la Banque asiatique de développement, indique l'amplitude et le succès de la mobilisation des diasporas hautement qualifiées de la Chine: l'estimation de la population des overseas Chinese professionals (OCP) dans le monde est de 1 million, avec de fortes concentrations en Amérique du

13 M. Brown, «Intellectual Diaspora Networks: Their Viability as a Response to Highly Skilled Emigration», Autrepart, n 22, 2002, pp. 167-178.

14 R. Barré, V. Hernandez, J.-B. Meyer et D. Vinck, Diasporas scientifiques/Scientific Diasporas, Paris, Editions IRD, 2003.

15 L. Lowell and S. Gerova, op. cit.

16 J.-B. Meyer and J.-P. Wattiaux, «Diaspora Knowledge Networks: Vanishing Doubts and Increasing Evidence», International Journal on Multicultural Societies, vol. 8, n 1, 2006, pp. 4-24.

17 S. Tobin and N. Sallee, «One Million Secret Agents: A report on Diaspora, Drains and Development», Kea (New Zealand Talent Community Abroad), 2006, disponible sur <http://www.keanewzealand. com/index.html>.

18 Biao Xiang, Promoting Knowledge Exchange through Diaspora Networks (The Case of People's Republic of China), Oxford, Centre on Migration, Policy and Society (COMPAS), University of Oxford, March 2005. 
Nord; on compte plus de 200 associations de ces OCP enregistrées par le State Council Overseas Chinese Affairs Office (OCAO); une politique délibérée, placée sous le slogan weiguo fuwu (signifiant «aider le pays»), a été mise en place à la fin des années 1990 afin de promouvoir les liens avec les talents de la diaspora $^{19}$; cinq ministères centraux et un grand nombre d'agences et de gouvernements provinciaux ainsi que des entités paraétatiques sont engagés dans des programmes et activités avec des expatriés hautement qualifiés; de courtes visites, des projets en collaboration, des cours d'éminents expatriés, des avis techniques ponctuels, des recrutements par des foires d'embauche ou des rencontres plus sélectives font partie des multiples échanges entre diaspora et communautés locales.

Le rôle des associations d'OCP, dont le nombre a crû durant ces dernières années, apparaît comme un facteur clé de l'expansion des liens. La formalisation de ces derniers, accompagnée par le soutien politique multipartite, dynamise les réseaux.

Le rôle de la diaspora dans le développement de l'industrie informatique indienne a fait récemment l'objet de nombreux commentaires. Tous les spécialistes convergent pour en reconnaître l'importance mais ne s'accordent pas sur la priorité à lui donner. Certains auteurs subordonnent l'apport des expatriés à un facteur local primordial ${ }^{20}$ - essentiellement la présence de main-d'oeuvre locale très qualifiée et bon marché - tandis que d'autres considèrent que l'intervention des associations d'Indiens de l'étranger aux Etats-Unis a été déterminante ${ }^{21}$.

De fait, la corrélation entre les développements de l'informatique indienne, la multiplication des associations de l'extérieur et l'intensification de leurs échanges avec le pays est frappante. Le suivi historique de certains de ces liens prouve le caractère déterminant du rôle de la diaspora. L'Inde figure comme le premier pays pour le nombre de réseaux de diasporas des savoirs œuvrant au développement de leur nation d'origine recensés sur Internet. De plus, à ces réseaux aux objectifs affirmés de contribution intellectuelle et technique en faveur du pays il faut ajouter toutes les associations professionnelles d'Indiens de l'étranger dont le but consiste à servir les plans de carrière, les créations d'entreprise, les liens d'affaires et les multiples mises en réseau des membres des communautés indiennes/asiatiques sur le territoire nord-américain. Pareille profusion d'acteurs et d'intermédiaires rend ce milieu extrêmement dense et fertile. Cette situation a son pendant en Inde même, où le gouvernement central et ceux des différents Etats, de même que les universités, instituts technologiques,

19 J.-B. Meyer, «Les diasporas de la connaissance: atout inédit de la compétitivité du Sud», Revue internationale et stratégique, $\mathrm{n}^{\circ}$ 55, 2004, pp. 69-76.

20 R. Lucas, op. cit. D. Kapur and J. McHale, Give Us Your Best and Brightest: The Global Hunt for Talent and Its Impact on the Developing World, Washington, DC, Center for Global Development, 2005.

21 A.L. Saxenian, Y. Motoyama, X. Quan and D.R. Wittenborn, Local and Global Networks of Immigrant Professionals in Silicon Valley, San Francisco, Public Policy Institute of California, 2002. B. Khadria et E. Leclerc, «Exode des emplois contre exode des cerveaux, les deux faces d'une même pièce?», Autrepart, numéro spécial, La migration des emplois vers le Sud, n 37, 2006, pp. 37-51. M. Warrier, «Temporary Mobility Schemes and the Indian Software Industry», conference paper, COMPAS Annual Conference «International Labour Migration: In Whose Interests?», Centre for Advanced Study in the Social Sciences, Oxford, 5-6 July 2006. E. Leclerc and J.-B. Meyer, «Knowledge Diasporas for Development: A Shrinking Space for Scepticism», Asian Population Studies, vol. $3, n^{\circ} 1,2007$, pp. 153-168. 
associations professionnelles, fédérations, commissions et chambres de commerce, sont très présents et actifs. Entre Inde et Amérique, une circulation continue d'agents humains et matériels nourrit les deux pôles migratoires avec des tâches et des objets complémentaires. L'interactivité dans ce milieu riche et diffus est assurée par un usage systématique et créatif de la communication médiatisée par ordinateur.

Pour résumer, les cas de l'Inde et de la Chine montrent l'importance d'une volonté politique. Les deux Etats manifestent clairement leur soutien aux initiatives de la diaspora, depuis vingt ans pour la Chine, qui a tôt laissé tomber ses préventions antérieures vis-à-vis des expatriés auparavant considérés comme suspects. L'Inde a eu de façon précoce, dès les années 1970, l'intuition que sa diaspora qualifiée pouvait représenter un atout et être récupérable. Toutefois, une stratégie de mobilisation effective n'a vu le jour qu'à partir du début du siècle et c'est aujourd'hui qu'elle se développe concrètement. Mais on constate que ces dispositions favorables du gouvernement central ne sont pas tout: l'interposition de multiples acteurs, sub- ou paraétatiques, privés ou non gouvernementaux, est cruciale. C'est cette gouvernance, politique médiatisée des acteurs, qui apparaît comme l'enseignement des cas indien et chinois.

L'existence des diasporas du savoir et leur activité ne posent plus de doute aujourd'hui. L'intérêt croissant qu'y prêtent les pays d'origine et d'accueil ainsi que les organisations internationales est certain. Cependant, les réussites de ces expériences en termes d'apport au développement varient grandement selon les pays et les régions. Le défi du jour est de mieux comprendre, en les comparant, ces divers cas et d'en tirer des enseignements pour les politiques de coopération ouvertes par ce nouveau type de relations. Sur un plan conceptuel, l'étude approfondie des diasporas contemporaines s'enrichit du cas particulier mais authentique de celles liées aux savoirs et aux personnes hautement qualifiées ${ }^{22}$. Ce type de diasporas introduit en effet une dimension intellectuelle et développementale non prise en compte par les typologies classiques portant sur des diasporas plus traditionnelles ${ }^{23}$ et renouvelle ainsi les questions sur les fondements de cette forme sociale aujourd'hui cruciale.

\section{Une modélisation pour le développement}

Afin de mieux expliquer les défaillances et les réussites des réseaux des diasporas du savoir et d'éclairer la politique à leur égard, nous avons effectué un examen systématique de leurs dynamiques. Le matériel empirique considérable réuni par une expertise collégiale (état de l'art) et différentes études de terrain ont permis de dégager un modèle interprétatif robuste ${ }^{24}$. Ce dernier permet de dépasser la référence anecdotique à des pratiques exemplaires mais particulières pour expliquer les fondements du succès ou de l'échec des pratiques expérimentées

22 S. Dufoix, Les diasporas, Que sais-je ?, n 3683, Paris, PUF, 2003. W. Berthomière et Ch. Chivallon (dir.), Les diasporas dans le monde contemporain, Paris, Karthala, Paris, 2006. C. Bordes-Benayoun et D. Schnapper, Diasporas et nations, Paris, Odile Jacob, 2006.

23 R. Cohen, Global Diasporas: An Introduction, London, Routledge, 1997.

24 R. Barré et al., op. cit. J. Brinkerhoff, op. cit. Y. Kuznetsov (ed.), Diaspora Networks and the International Migration of Skills: How Countries Can Draw on Their Talents Abroad, Washington, DC, World Bank Institute, 2006. 
par différents réseaux depuis quinze ans. Le modèle est basé sur la théorie de l'acteur-réseau et sur le processus de traduction, issus tous deux de la sociologie de l'innovation. Ce processus se décompose en quatre opérations typiques des réseaux d'innovation et que l'on peut appliquer de façon pertinente aux réseaux diasporiques : la problématisation, la mobilisation, l'enrôlement et l'intéressement ${ }^{25}$.

En résumé, pour que réussisse une innovation, ses créateurs (scientifiques, entrepreneurs, département de recherche et développement, etc.) doivent reformuler la question ou le problème dans des termes et des conditions adéquats, obtenir l'intérêt des entités susceptibles de choisir le nouveau chemin, enrôler les alliés (humains et non humains) qui vont définir la nouvelle norme, mobiliser et canaliser les ressources et les acteurs sur ces nouveaux axes, les rendant ainsi progressivement irréversibles.

Le processus d'innovation n'est pas le fruit d'une logique où s'imposerait naturellement un artefact aux qualités intrinsèquement supérieures; il s'agit d'une lutte entre, d'une part, les entités et les réseaux existants et, d'autre part, ceux qui cherchent à obtenir pertinence et crédibilité et à s'établir durablement. Par conséquent, à bien des égards, une innovation signifie la dissociation des éléments d'une configuration précédente, en captant les acteurs et en mettant en place un réseau dans lequel ceux-ci tiennent ensemble d'une nouvelle manière. A travers la problématisation, la mobilisation, l'enrôlement et l'intéressement, il y a donc détournement des éléments précédents et de leurs associations au profit de nouveaux consortia.

Cette approche correspond à la situation des diaspora knowledge networks (DKN). L'expatrié est un acteur-réseau, condensant en sa personne ses connexions (capital social). Le but des réseaux DKN est de capturer cet acteurréseau et de le détourner vers le pays d'origine, qui est lui-même, au moins potentiellement, un consortium de réseaux. En ce sens, afin de réaliser le processus de traduction, le réseau DKN doit effectuer les opérations de problématisation, de mobilisation, d'enrôlement et d'intéressement. De fait, le constat empirique révèle que la plupart des DKN sont parvenus à réaliser les trois premières de ces opérations.

\section{Problématisation}

Le problème de la circulation des compétences a été reformulé avec succès, par la substitution d'une conception constructive (le brain gain) à une conception négative (l'exode des cerveaux) au cours des deux décennies passées. Avec ce changement s'est imposée une vision dans laquelle l'éventuelle reconnexion est devenue une solution viable et prometteuse. Aujourd'hui, aucun pays ni aucune organisation internationale ne rejetterait la possibilité de travailler en réseau avec

25 M. Callon, «Some Elements for a Sociology of Translation: Domestication of the Scallops and the Fishermen of St-Brieuc Bay », in J. Law (ed.), Power, Action and Belief: A New Sociology of Knowledge?, London, Routledge and Kegan Paul, 1986, pp. 196-229. B. Latour, Science in Action: How to Follow Scientists and Engineers through Society, Milton Keynes, Open University Press, 1987. J. Law and J. Hassard (eds.), Actor Network Theory and After, London, Blackwell Publishing, 1999. B. Latour, Reassembling the Social: An Introduction to Actor-Network Theory, Oxford; New York, Oxford University Press, 2005. 
les expatriés d'une manière systématique, ce qui ouvre des perspectives pour de nombreux DKN. Bien des expatriés ont implicitement ou explicitement reconnu leur contribution potentielle à leur pays d'origine. Ils ne se considèrent plus comme inaccessibles et se rendent disponibles pour des projets gérés par leurs homologues dans les pays d'origine. Il s'agit d'une posture concrète et pragmatique de brain gain, au lieu de l'ancien schéma d'une expatriation permanente et complète qui régnait auparavant dans l'esprit de ces personnes. Des entretiens personnels recomposant l'historique individuel de la migration montrent cette reformulation de la mobilité comme une expérience de vie opposée à la rupture définitive avec le lieu de naissance. Un changement crucial dans cette reformulation a été l'introduction de la communication par Internet, comme reconstruction de la continuité entre des espaces et des séquences de temps séparés.

\section{Mobilisation}

Dans tous les cas où de réelles tentatives de mise en place de réseaux ont été impulsées, elles ont réussi à rassembler un grand nombre d'expatriés. L'ampleur de la mobilisation varie considérablement d'un réseau à l'autre, de pays à pays, mais le message d'appel s'avère séduisant pour beaucoup. Ce message a généralement été diffusé par des listes de courrier électronique à partir de contacts personnels ou institutionnels. Fait intéressant, de nombreux expatriés avouent que, préalablement à un tel appel, ils étaient plutôt réticents à toute initiative nationale, publique ou privée. Le fait qu'elle se fonde sur des raisons et des intérêts professionnels et intellectuels semble avoir stimulé les processus de mobilisation, alors que les canaux politiques ou patriotiques apparaissent plus dissuasifs et suspects. Le nouveau média, Internet, est neutre sur ces aspects et, dans ce contexte particulier, a permis la reconnexion sur de nouvelles bases.

\section{Enrôlement}

Les nombreux réseaux identifiés (voir plus haut) révèlent un degré élevé d'enrôlement formel dans des structures associatives. Les expatriés acceptent de devenir membres d'une entreprise collective avec un but explicite dans lequel ils jouent un rôle défini. L'examen des sites Internet de ces réseaux montre qu'un certain nombre d'associations exposent les coordonnées personnelles et professionnelles de leurs membres. Elles sont stockées dans des bases de données et constituent des composants du réseau, dont ces membres ont accepté de servir l'objectif, devenant par là même accessibles aux acteurs anonymes qui pourraient atteindre le réseau par Internet avec leurs propres motifs. Les acteurs sont donc «ponctualisés» dans le réseau, c'est-à-dire que ce dernier les totalise en son sein et devient le point de passage obligé à des tiers pour y accéder ${ }^{26}$.

Le matériel empirique démontre ainsi clairement que la problématisation, la mobilisation et l'enrôlement ont été réalisés fréquemment dans l'option diaspora en général et par de nombreux DKN individuellement. Cependant, il est une opération pour laquelle les résultats ont été la plupart du temps beaucoup moins satisfaisants : l'intéressement. Et de l'intéressement dépend la durabilité... 


\section{L'intéressement}

L'intéressement est ce qui lie durablement l'acteur au réseau. Au-delà de simples mesures d'incitation susceptibles de générer des motivations psychologiques, il recouvre tous les intermédiaires attachant l'acteur à un réseau particulier. Inter-esse, en latin, signifie «être entre». Les dispositifs d'intéressement sont ceux qui se tiennent entre les acteurs et nourrissent l'action collective à travers laquelle ils s'associent. Ils partent du principe que l'acteur évolue dans un environnement compétitif: l'intéressement est ce qui lui fait choisir et maintenir son choix pour un réseau, parmi d'autres connexions possibles.

Dans les DKN, comme dans les réseaux d'innovation en général, les dispositifs d'intéressement peuvent être très divers: programmes, financements, invitations, réunions, récompenses, contrats, information, etc. Comme il s'agit d'un groupe d'acteurs physiquement dispersés, les technologies de l'information et de la communication (TIC) jouent un rôle crucial parmi ces dispositifs. Elles ont le pouvoir d'extraire l'individu expatrié de ses réseaux locaux quotidiens et de l'insérer dans ceux du pays d'origine qui lui conviennent. Toutefois, cela n'a de sens qu'à des fins d'activité concrète, puisque la participation au réseau n'est pas motivée par la communication per se mais par l'action orientée vers le développement dans le pays d'origine. Cela explique, par exemple, la lassitude exprimée par les membres du réseau Caldas à l'égard des listes passives de diffusion générale saturant leur boîte de réception, et par conséquent l'importance de concevoir des dispositifs interactifs ad hoc. L'expérience prouve que les TIC ont le pouvoir d'attirer l'attention des expatriés sur des actions dans les réseaux du pays d'origine. Mais sans action, ce pouvoir attractif s'évanouit rapidement.

Cependant, il est un trait spécifique des réseaux DKN - à la différence des réseaux traditionnels d'innovation - dans les procédures d'intéressement et qui peut expliquer certaines de leurs lacunes en la matière. A l'opposé des réseaux classiques d'innovation, tels que ceux présentés dans l'approche théorique qui précède, les DKN ne visent pas à capter exclusivement et définitivement les acteurs-réseaux représentés par les expatriés. Ils essaient, au contraire, de les associer de manière seulement ponctuelle, de telle sorte que ces acteurs puissent garder leurs connexions multiples antérieures. Séparer l'expatrié de ses précieux réseaux est à l'opposé des objectifs fondés sur les DKN, à la différence des processus traditionnels d'innovation. Le membre d'un réseau DKN est, par définition, le pont entre ses propres réseaux dans le pays d'accueil et ceux des pays d'origine. Ce régime coopératif (et non compétitif, comme dans les autres réseaux d'innovation) est théoriquement possible, et en fait parfois réussi, tel dans les cas asiatiques évoqués précédemment, mais la tension existe bel et bien. Elle peut déboucher sur un retrait de l'acteur si les dispositifs d'intéressement ne sont pas suffisamment forts, comme un certain nombre de cas le démontrent.

L'expatrié est généralement bien implanté dans ses réseaux, socioprofessionnels et autres, dans le pays d'accueil (voir plus haut). Ils génèrent localement de forts dispositifs d'intéressement qui ont tendance à tenir l'acteur à distance de toute association faiblement concurrentielle proposée depuis un lieu périphérique. Par exemple, comment un éminent généticien, menant des projets internationaux depuis le Royaume-Uni, pourrait-il s'intéresser à établir et maintenir des liens 
avec ses pairs dans son pays natal, le Bangladesh, s'il n'y a pas de demande exprimée et soigneusement relayée, si aucune perspective de débouché n'est donnée, aucun appui concret envisagé, aucune information précise fournie? En dépit de sa prise de conscience des problèmes du pays (problématisation), de sa motivation personnelle (mobilisation) et de son implication formelle sanctionnée par son inscription comme membre de l'association locale d'expatriés (enrôlement), il tendra à abandonner le réseau, lentement ou rapidement. Il cherchera même souvent à se prémunir contre de nouvelles sollicitations de la part de ce dernier, pour éviter d'inutiles perturbations dans son travail quotidien, lorsqu'il aura constaté que son engagement n'est pas contrebalancé par des investissements de l'autre côté.

C'est cette défaillance répétée de l'intéressement qui a détourné de nombreux acteurs des réseaux DKN. Elle met en jeu la nécessité de combler ce vide, en créant les médiations nécessaires à l'action, et pointe l'importance des gouvernances de coopération à mettre en place à cet effet.

\section{Conclusion}

L'émergence des diasporas scientifiques et techniques invalide les approches traditionnelles en termes de fuite des cerveaux et consacre l'avènement du paradigme circulatoire dans l'étude des migrations internationales. Il reste à prendre acte de cette configuration nouvelle, en cessant de reproduire les schémas anciens des asymétries Nord-Sud. Ce nouvel état d'esprit devrait pénétrer tant la conception de la recherche que celle des politiques. Il faut transposer les schèmes de l'approche transnationale - dont relève la diaspora - aux politiques migratoires et de coopération. Recourir aux cloisonnements du passé pour interpréter les nouvelles logiques à l'œuvre ou tenter de bloquer ou contraindre les circulations vitales de la mondialisation, c'est contredire l'histoire et risquer d'y introduire de coûteuses ruptures.

Notre conclusion débouche sur une proposition: aller jusqu'au bout des nouvelles logiques circulatoires en les accompagnant par des formes inédites de gouvernance, construites avec l'apport de recherches originales. Il ne s'agit guère de penser en termes mécanistes de gestion des flux, ouvrant les vannes de façon plus ou moins sélective, comme cela est généralement le cas, la politique suivant en cela de façon simpliste une approche quantitative. Il faut tirer parti des mobilités exprimées, en les accompagnant de dispositifs de réalisation. Si l'on constate que des individus et des groupes expatriés se constituent, s'autoorganisent et visent à opérer des transferts valorisant le patrimoine du pays d'accueil et bénéficiant au pays d'origine, il y a tout lieu de cultiver ces liens utiles aux deux pôles de la migration. Cette culture du lien, fondée sur la reconnaissance des réseaux migratoires et leurs enracinements locaux multiples, doit prévaloir sur l'approche dichotomique assimilationniste ou communautariste.

Promouvoir et développer cette culture n'est pas chose facile et la pratique de ces liens s'avère difficile. En effet, si les initiatives qui ont pressenti les apports possibles de la diaspora sont nombreuses, plus rares sont celles qui sont parvenues à les réaliser de façon notable. L'analyse de réseau permet de constater que la plupart des défaillances se situent dans l'opération d'intéressement, constitutive de 
ces liens. Sans attachement soutenu et durable des acteurs aux processus d'association entre diaspora et pays d'origine, le développement de ce dernier ne peut bénéficier de cette ressource constituée. Cet intéressement ne peut se réaliser sans investissements. Cependant, ceux-ci sont moins de nature économique que sociale au sens large, puisqu'ils consistent à susciter des médiations multiples à de nombreux niveaux, à l'instar des expériences chinoises et indiennes évoquées précédemment. Ces investissements relationnels sont mis en évidence par le modèle d'acteur-réseau adopté pour l'analyse systématique des multiples cas identifiés. La politique peut concrètement s'en inspirer pour une gouvernance des réseaux, sur le canevas du soft power plutôt que du dirigisme ou du laisserfaire $^{27}$. La modélisation revêt alors non pas un caractère normatif, mais une dimension suggestive.

\section{Références bibliographiques}

Badie, B., R. Brauman, E. Decaux, G. Devin et C. Wihtol de Wenden, Pour un autre regard sur les migrations. Construire une gouvernance mondiale, Paris, La Découverte, 2008.

Barré, R., V. Hernandez, J.-B. Meyer et D. Vinck, Diasporas scientifiques/Scientific Diasporas, Paris, Editions IRD, 2003.

Berthomière, W. et Ch. Chivallon (dir.), Les diasporas dans le monde contemporain, Paris, Karthala, Paris, 2006.

Bordes-Benayoun, C. et D. Schnapper, Diasporas et nations, Paris, Odile Jacob, 2006.

Brinkerhoff, J., «Diasporas, Mobilization Factors and Policy Options », in C. Wescott and J. Brinkerhoff (eds.), Converting Migration Drains into Gains: Harnessing the Resources of Overseas Professionnals, Manila, Asian Development Bank, 2006, pp. 127-153.

Brown, M., «Intellectual Diaspora Networks: Their Viability as a Response to Highly Skilled Emigration», Autrepart, $\mathrm{n}^{\circ}$ 22, 2002, pp. 167-178.

Callon, M., «Some Elements for a Sociology of Translation: Domestication of the Scallops and the Fishermen of St-Brieuc Bay », in J. Law (ed.), Power, Action and Belief: A New Sociology of Knowledge?, London, Routledge and Kegan Paul, 1986, pp. 196-229.

Cohen, R., Global Diasporas: An Introduction, London, Routledge, 1997.

Docquier, F. and A. Marfouk, Measuring the International Mobility of Skilled Workers (1990-2000), World Bank Policy Research Working Paper, nº 3381, Washington, DC, World Bank, 2004.

Dubar, C., La socialisation: construction des identités sociales et professionnelles, Paris, Armand Colin, 1991.

Dufoix, S., Les diasporas, Que sais-je ?, nº 3683, Paris, PUF, 2003.

Dumont, J.-C. and G. Lemaître, «Beyond the Headlines: New Evidence on the Brain Drain», La Revue économique, vol. 56, $\mathrm{n}^{\circ}$ 6, 2005, pp. 1275-1299.

Findlay, A.M. and E. Stewart, Skilled Labour Migration from Developing Countries: Annotated Bibliography, International Migration Papers, $n^{\circ}$ 55, Geneva, International Labour Office, 2002.

Gaillard, J. and A.-M. Gaillard, «Can the Scientific Diaspora Save African Science?», 2003, disponible sur le site du Science and Development Network (Scidev), <http://www.scidev.net>.

Gaillard, J. et A.-M. Gaillard, Les enjeux des migrations scientifiques internationales. De la quête du savoir à la circulation des compétences, Paris, L'Harmattan, 1997.

Gamlen, A., The Brain Drain Is Dead: Long Live the New Zealand Diaspora, COMPAS Working Paper, $\mathrm{n}^{\mathrm{o}}$ 10, Oxford, Centre on Migration, Policy and Society (COMPAS), Oxford University, 2005.

Gellner, E., Nations and Nationalism, Oxford, Basil Blackwell, 1983.

Granés, J. y A. Morales, «Las Potencialidades y Limitaciones de la Red Caldas de Investigadores Colombianos en el Exterior: Los Proyectos Internacionales Conjuntos: Un Estudio de Casos », in J. Charum y J.-B. Meyer (coordinadores), Hacer Ciencia en un Mundo Globalizado: La Diáspora Científica Colombiana en Perspectiva, Santafé de Bogotá, TM Editores, 1998.

27 B. Badie, R. Brauman, E. Decaux, G. Devin et C. Wihtol de Wenden, Pour un autre regard sur les migraions. Construire une gouvernance mondiale, Paris, La Découverte, 2008. 
Granés, J. and J.-B. Meyer, «Globalization of the National Scientific Community through Electronic List: Lessons and Prospects from a Case Study in Colombia», in J. Charum and J.-B. Meyer (eds.), International Scientific Migrations Today, CD-Rom, Paris, Editions IRD, 2000.

Guo, Y., «How Can the Chinese Intellectual Diasporas Bridge their Host and Home Countries as well as Help Their Home Country Integrate into the International Community », in R. Barré, V. Hernandez, J.-B. Meyer et D. Vinck, Diasporas scientifiques/Scientific Diasporas, Paris, Editions IRD, 2003.

Johnson, J., «S\&T Resources and Programs in the United States for Networking with Developing Countries », in R. Barré, V. Hernandez, J.-B. Meyer et D. Vinck, Diasporas scientifiques/Scientific Diasporas, Paris, Editions IRD, 2003.

Kapur, D. and J. McHale, Give Us Your Best and Brightest: The Global Hunt for Talent and Its Impact on the Developing World, Washington, DC, Center for Global Development, 2005.

Khadria, B. et E. Leclerc, «Exode des emplois contre exode des cerveaux, les deux faces d'une même pièce? ", Autrepart, numéro spécial, La migration des emplois vers le Sud, n 37, 2006, pp. 37-51.

Kuznetsov, Y. (ed.), Diaspora Networks and the International Migration of Skills: How Countries Can Draw on Their Talents Abroad, Washington, DC, World Bank Institute, 2006.

Latour, B., Reassembling the Social: An Introduction to Actor-Network Theory, Oxford; New York, Oxford University Press, 2005.

Latour, B., Science in Action: How to Follow Scientists and Engineers through Society, Milton Keynes, Open University Press, 1987.

Law, J. and J. Hassard (eds.), Actor Network Theory and After, London, Blackwell Publishing, 1999.

Leclerc, E. and J.-B. Meyer, «Knowledge Diasporas for Development: A Shrinking Space for Scepticism», Asian Population Studies, vol. 3, n 1, 2007, pp. 153-168.

Lowell, L., Skilled Labour Migration from Developing Countries: Annotated Bibliography, International Migration Papers, $n^{\circ}$ 56, Geneva, International Labour Office, 2002.

Lowell, L. and S. Gerova, Diasporas and Economic Development: State of Knowledge, Washington, DC, World Bank, 2004.

Lucas, R., International Migration Regimes and Economic Development, Stockholm, Expert Group on Development Issues (EGDI), 2004.

Meyer, J.-B., «Savoirs, diasporas et identités projectives », Hermès, no 40, 2004, pp. 350-354.

Meyer, J.-B., «Les diasporas de la connaissance: atout inédit de la compétitivité du Sud», Revue internationale et stratégique, $\mathrm{n}^{\circ} 55,2004, \mathrm{pp} .69-76$.

Meyer, J.-B. and J.-P. Wattiaux, «Diaspora Knowledge Networks: Vanishing Doubts and Increasing Evidence», International Journal on Multicultural Societies, vol. 8, nº 1, 2006, pp. 4-24.

Meyer, J.-B., D. Kaplan and J. Charum, «Scientific Nomadism and the New Geopolitics of Knowledge», International Social Sciences Journal/Revue internationale des sciences sociales, $\mathrm{n}^{\circ}$ 168, 2001, pp. 341-354.

Meyer, J.-B. et al., «Turning Brain Drain into Brain Gain: The Colombian Experience of the Diaspora Option», Science, Technology and Society, vol. 2, n² 2, 1997, pp. 285-317.

Saxenian, A.L., Y. Motoyama, X. Quan and D.R. Wittenborn, Local and Global Networks of Immigrant Professionals in Silicon Valley, San Francisco, Public Policy Institute of California, 2002.

Tobin, S. and N. Sallee, «One Million Secret Agents: A report on Diaspora, Drains and Development», Kea (New Zealand Talent Community Abroad), 2006, disponible sur <http://www.keanewzealand.com/ index.html>.

Warrier, M., «Temporary Mobility Schemes and the Indian Software Industry », conference paper, COMPAS Annual Conference «International Labour Migration: In Whose Interests?», Centre for Advanced Study in the Social Sciences, Oxford, 5-6 July 2006.

Wescott, C., «Promoting Knowledge Exchange through Diasporas», conference paper, G-20 workshop on «Demographic Challenges and Migration», Sydney, 27-28 August 2005.

Xiang, Biao, Promoting Knowledge Exchange through Diaspora Networks (The Case of People's Republic of China), Oxford, Centre on Migration, Policy and Society (COMPAS), University of Oxford, March 2005 